\title{
Collaborative Location Privacy with Rational Users
}

\author{
Francisco Santos, Mathias Humbert, Reza Shokri, and Jean-Pierre Hubaux \\ School of Computer and Communication Sciences, EPFL, Switzerland \\ firstname.lastname@epfl.ch
}

\begin{abstract}
Recent smartphones incorporate embedded GPS devices that enable users to obtain geographic information about their surroundings by providing a location-based service (LBS) with their current coordinates. However, LBS providers collect a significant amount of data from mobile users and could be tempted to misuse it, by compromising a customer's location privacy (her ability to control the information about her past and present location). Many solutions to mitigate this privacy threat focus on changing both the architecture of location-based systems and the business models of LBS providers. MobiCrowd does not introduce changes to the existing business practices of LBS providers, rather it requires mobile devices to communicate wirelessly in a peer-to-peer fashion. To lessen the privacy loss, users seeking geographic information try to obtain this data by querying neighboring nodes, instead of connecting to the LBS. However, such a solution will only function if users are willing to share regional data obtained from the LBS provider. We model this collaborative location-data sharing problem with rational agents following threshold strategies. Initially, we study agent cooperation by using pure game theory and then by combining game theory with an epidemic model that is enhanced to support threshold strategies to address a complex multi-agent scenario. From our game-theoretic analysis, we derive cooperative and non-cooperative Nash equilibria and the optimal threshold that maximizes agents' expected utility.
\end{abstract}

\section{Introduction}

Today's smartphones are often equipped with GPS devices that enable their users to obtain contextual information about their surroundings, such as the location of the nearest supermarket, without needing to ask directions from other people. To obtain such contextual information, users normally query a location-based service (LBS), such as Google Maps (http://maps.google.com) that, given a current position, can provide detailed information about points-ofinterest in the region and stepwise instructions to reach a particular destination. The downside of using an LBS system is the possible loss of location privacy [5] 10, 12], defined as the ability for a user to control how, where, and when information about her current and past location is used and by whom [1].

MobiCrowd [14] mitigates the loss in location privacy by assuming users carry location-aware wireless devices capable of peer-to-peer communication, through 
which they can share regional data and, in this way, reduce the fraction of queries dispatched to the LBS. MobiCrowd devices are equipped with a mobile proxy that stores the results of LBS queries in a buffer. When a user issues a new query, the mobile proxy scans the buffer for the information. If the query cannot be answered by the local cache, it is broadcast to peer devices within range. Should these peers be unable or unwilling to answer the query, the device finally prompts the LBS server. The main advantage of this new scheme is that it helps protect users' location privacy and requires no changes to the current business practices of LBS providers and only minimal changes to the architecture of conventional location-based services.

Our goal is to learn whether the overall level of cooperation amongst users is sufficiently high for a solution such as MobiCrowd to work. For this purpose, we model the problem by using game theory 2,13 . This discipline provides a rich set of analytical tools through which researchers study the interaction between agents as decision-makers, notably in the context of cooperation in wireless communications [7] and in location privacy [1, 4, 8, 9, [11. Combining game theory with epidemic models, such as the susceptible-infected-removed (SIR) model [6], is a practical approach to studying strategic behavior for large populations of agents. This method is used in $[3$ to explore why rational individuals might preclude the eradication of a vaccine-preventable disease by weighing the risks of vaccination and infection; and in [15] to study how the investment in security by self-interested agents affects the propagation of a computer network infection.

In our game-theoretic model, we represent users as agents who follow threshold strategies. We assume that users are rational, meaning that they have knowledge of their actions, reason about uncertainty, have clear preferences expressed through a utility function, and choose actions in their own self-interest by maximizing this utility function [13]. Using this model, we define two infinitely repeated games of imperfect information. In the first game, we study an elementary, two-agent interaction and use pure game theory to derive Nash and Pareto optimal equilibria. In the second, we analyze a complex, multi-agent interaction by using a modified version of the epidemic model in [14 to support threshold strategies and derive the optimal threshold that maximizes agents' payoffs.

\section{System Model}

In this section we define a game-theoretic framework from which we scrutinize the MobiCrowd architecture. Consider an agent $i$ confined to a single region. As it interacts with other agents in the region and the LBS, it switches between three distinct roles: seeker (K), informed (I), and removed (R) 14. Seekers try to obtain regional data by querying other peers or, ultimately, the LBS server. Informed agents have data on the region and accept to spread this information according to a threshold strategy; they become removed once the data on their mobile proxy expires. Finally, removed agents are not interested in obtaining regional data but can become information seekers later in the game. The set of roles that each agent may have is defined as follows: $\Pi_{R}=\{K, I, R\}$. We illustrate the interaction between the three agent roles in Figure 1 . 

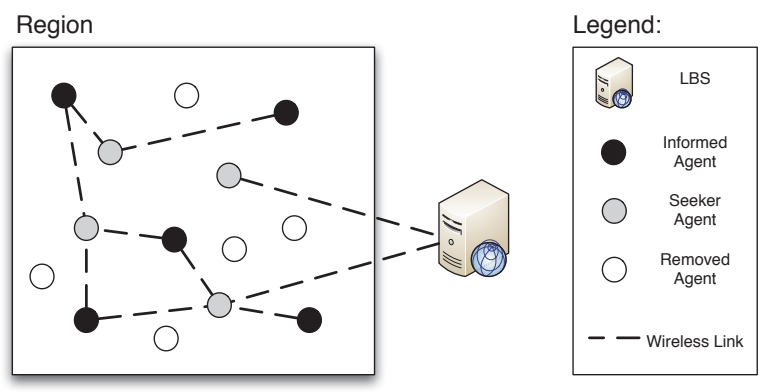

Fig. 1. Operating principle of MobiCrowd. Seekers attempt to obtain data on this region through informed agents or the LBS using wireless communication links. Removed agents do not have any regional data and do not want to obtain such information, abstaining from any interaction with either seekers or informed agents.

As agent $i$ communicates with its peers, it records (i) the number of times it received data from other agents, $\mathrm{rc}_{i}(t)$, and (ii) the number of times it answered queries from its peers, $\operatorname{tr}_{i}(t)$, up to and excluding time $t$, where $\operatorname{rc}_{i}(0)=\operatorname{tr}_{i}(0)=$ 0 . The goal is to define an agent's cumulative cooperation effort: the total number of times an agent receives help in excess of the amount of times it shared data with a peer. An agent uses this value to decide whether to cooperate or defect when receiving queries from its peers. Let $\phi_{i}(t)$ denote the cumulative cooperation effort of agent $i$, defined as follows:

$$
\phi_{i}(t)=\mathrm{rc}_{i}(t)-\operatorname{tr}_{i}(t) .
$$

Further, let the set $\Pi_{\phi}$ equal the range of the cumulative cooperation effort: $\Pi_{\phi}=$ $\left\{\phi^{\min }, \ldots, 0, \ldots, \phi^{\max }\right\}$, where $\phi^{\text {min }}$ and $\phi^{\text {max }}$ are, respectively, the minimum and maximum bounds for $\phi_{i}(t)$.

There are two variables that define the state of an agent in the system: (i) its current role and (ii) its cumulative cooperation effort. Hence, by combining these two variables, we can define the full set of agent states. Let $\mathcal{S}$ denote the set of agent states, computed as the cartesian product of $\Pi_{R}$ and $\Pi_{\phi}: \mathcal{S}=\Pi_{R} \times \Pi_{\phi}$. We adopt the following notation to refer to each state: $\mathcal{X}_{\phi}$, where $\mathcal{X}$ represents one of the three possible roles and $\phi$ is the cumulative cooperation effort. Additionally, we express the fraction of agents in each state at time $t$ as $X_{\phi}(t)$.

An agent $i$ decides to share regional facts with seekers according to a threshold strategy $s_{i}(t)$. It cooperates with a seeker if (i) it is informed and (ii) its cumulative cooperation effort, $\phi_{i}(t)$, is above a common threshold $\alpha$ that expresses the amount of sharing agents accept to perform before expecting some aid in return; lowering $\alpha$ improves an agent's capacity to collaborate. If either condition is false, the agent defects:

$$
s_{i}(t)= \begin{cases}\text { cooperate } & \text { if } \phi_{i}(t)>\alpha \wedge i \in \mathcal{I}_{\phi_{i}(t)}, \\ \text { defect } & \text { otherwise }\end{cases}
$$


As in [14, we assume that agents share a single kind of contextual information. To consider multiple types of regional data, we can apply the same system model to each type of data separately; hence, no loss of generality results from this simplifying assumption.

In our work, we consider a payoff model that reflects the costs and benefits experienced by MobiCrowd users as they interchange data with peers and connect to the LBS. Consider a simple interaction between a seeker $i$, an informed agent $j$, and the LBS. When $i$ prompts $j$ and $j$ cooperates, $i$ receives a benefit of $b_{\text {inf }}$, expressing the agent's information gain, and $j$ incurs a communication cost of $c_{\text {com }}$ for sharing data. If $j$ defects it receives no payoff and $i$ must seek help from the LBS, earning a lesser payoff of $b_{\text {inf }}-c_{\text {srv }}$, where $c_{\text {srv }}$ denotes the privacy loss implied when communicating with the location-based service. The utility of agent $i$ at time $t$ is defined as the difference between the benefit obtained, $b_{i}(t)$, and the cost incurred, $c_{i}(t)$, at time $t$ :

$$
u_{i}(t)=b_{i}(t)-c_{i}(t) .
$$

According to the operating principle of MobiCrowd [14, users that seek geographic data query nearby informed peers to avoid the privacy threat posed by the LBS; hence, we assume that the LBS privacy cost $\left(c_{\text {srv }}\right)$ exceeds the peer-to-peer communication cost $\left(c_{\text {com }}\right)$. As users ultimately endeavor to become informed, we further assume that the benefit of acquiring data $\left(b_{\text {inf }}\right)$ tops the privacy and communication costs: $0 \leq c_{\mathrm{com}}<c_{\mathrm{srv}}<b_{\mathrm{inf}} \leq 1$.

In infinitely repeated games, agents lack knowledge of the precise game duration, thus, they value present payoffs more than future rewards. To capture this fact, we define the aggregate discounted reward of an agent for two distinct cases: discrete time and continuous time games. In the discrete case, the payoff of an agent $i$ is observed at several discrete time instances or game stages and the aggregate discounted reward is the sum of its payoff in the immediate stage game, plus the sum of aggregate rewards discounted by a constant $\delta \in] 0,1[$.

Definition 1. Given an infinite sequence of payoffs $u_{i}(0), u_{i}(1), \ldots$ for agent $i$, and a discount factor $\delta$ with $0<\delta<1$, the aggregate discounted reward of $i$ in a discrete time game is: $\sum_{t=0}^{\infty} \delta^{t} u_{i}(t)=\sum_{t=0}^{\infty} \delta^{t}\left(b_{i}(t)-c_{i}(t)\right)$.

Additionally, we consider a continuous time game, in which case the aggregate discounted reward is given by the integral of an agent's payoff for the duration of the game, discounted by a constant $\delta \in] 0,1[$.

Definition 2. Given the utility function $u_{i}(t)$ for agent $i$ and a discount factor $\delta$ with $0<\delta<1$, the aggregate discounted reward of $i$ in a continuous time game is: $\int_{0}^{\infty} \delta^{t} u_{i}(t) d t=\int_{0}^{\infty} \delta^{t}\left(b_{i}(t)-c_{i}(t)\right) d t$.

\section{Two-Agent Game}

We first model the problem as an infinitely repeated two-agent game of imperfect information and we constrain both agents to a single map region. To avoid this 
Table 1. List of Symbols

\begin{tabular}{|c|c|}
\hline \multicolumn{2}{|c|}{ Symbol Description } \\
\hline$n$ & size of the agent population \\
\hline$\phi_{i}(t)$ & cumulative cooperation effort of agent $i$ \\
\hline $\mathrm{rc}_{i}(t)$ & number of times agent $i$ received data up to and excluding time $t$ \\
\hline $\operatorname{tr}_{i}(t)$ & number of times agent $i$ shared data up to and excluding time $t$ \\
\hline$s_{i}(t)$ & pure strategy followed by agent $i$ at time $t$ \\
\hline$\alpha$ & $\begin{array}{l}\text { threshold value used in } s_{i}(t) \text { to trigger cooperative and non- } \\
\text {-cooperative agent behavior }\end{array}$ \\
\hline$\alpha^{\mathrm{opt}}$ & common threshold value that maximizes agents' expected payoff \\
\hline$u_{i}(t)$ & agent $i$ 's payoff at time $t$ \\
\hline$b_{i}(t)$ & agent $i$ 's benefit (if any) at time $t$ \\
\hline$c_{i}(t)$ & agent $i$ 's cost (if any) at time $t$ \\
\hline$b_{\text {inf }}$ & payoff for obtaining regional data from a peer or the LBS \\
\hline$c_{\mathrm{com}}$ & communication cost of sharing regional data with a peer \\
\hline$c_{\mathrm{srv}}$ & privacy cost incurred when calling the LBS server \\
\hline$\delta$ & discount factor used to calculate agents' aggregate payoffs \\
\hline$U_{i}$ & agent $i$ 's expected aggregate discounted payoff \\
\hline$\widehat{U}$ & estimate of an agent's expected aggregate discounted payoff \\
\hline$U_{\text {total }}$ & total discounted payoff of the game \\
\hline$\widehat{U}_{\text {total }}$ & estimate of the game's total discounted payoff \\
\hline $\mathrm{rc}_{\text {total }}$ & total rate at which seekers receive data from informed peers \\
\hline$\widehat{\mathrm{rc}}_{\text {total }}$ & $\begin{array}{l}\text { estimate of the total rate at which seekers receive data from } \\
\text { informed peers }\end{array}$ \\
\hline $\operatorname{tr}_{\text {total }}$ & total rate at which informed agents share data with seekers \\
\hline$\widehat{\operatorname{tr}}_{\text {total }}$ & $\begin{array}{l}\text { estimate of the total rate at which informed agents share data } \\
\text { with seekers }\end{array}$ \\
\hline$\widehat{\mathrm{sv}}_{\text {total }}$ & $\begin{array}{l}\text { estimate of the total rate at which seekers receive data from the LBS } \\
\text { set of game states }\end{array}$ \\
\hline$\Pi_{\phi}$ & range of the cumulative cooperation effort: $\left\{\phi^{\mathrm{min}}\right.$, \\
\hline$\Pi_{R}$ & set of possible agent roles: $\{K, I, R\}$ \\
\hline $\mathcal{K}_{\phi}$ & $\begin{array}{l}\text { an agent } i \text { is in this game state at time } t \text { if it wants to obtain } \\
\text { regional data and } \phi_{i}(t)=\phi\end{array}$ \\
\hline$K_{\phi}(t)$ & fraction of agents in state $\mathcal{K}_{\phi}$ at time $t$ \\
\hline $\mathcal{I}_{\phi}$ & $\begin{array}{l}\text { an agent } i \text { is in this game state at time } t \text { if it has regional data } \\
\text { and } \phi_{i}(t)=\phi\end{array}$ \\
\hline$I_{\phi}(t)$ & fraction of agents in state $\mathcal{I}_{\phi}$ at time $t$ \\
\hline $\mathcal{R}_{\phi}$ & $\begin{array}{l}\text { an agent } i \text { is in this game state at time } t \text { if it has no regional data, } \\
\text { does not wish to obtain this data, and } \phi_{i}(t)=\phi\end{array}$ \\
\hline$R_{\phi}(t)$ & fraction of agents in state $\mathcal{R}_{\phi}$ at time $t$ \\
\hline
\end{tabular}


privacy threat, agents 1 and 2 want to obtain information about this region by querying their partner, instead of calling the LBS. In the beginning, no agent has the desired data, consequently one agent must call the LBS. The data retrieved from the LBS expires after one game epoch, at which time it is deleted from the agents' mobile proxy. At the start of each stage, exactly one agent - chosen uniformly at random - will be responsible for querying the server. Upon querying the LBS, an agent $i$ becomes informed and follows a threshold strategy to decide whether to cooperate and share data with its peer $j$ or defect by refusing to share, according to the strategy function $s_{i}(t)$ (2).

When agent $i$ cooperates at time $t$, i.e. $s_{i}(t)=$ cooperate, $i$ incurs a commu-

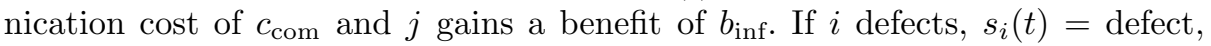
it obtains no payoff and $j$ must call the LBS, earning $b_{\text {inf }}-c_{\text {srv }}$. The utility function, shared by both agents, is defined succinctly as follows:

$$
u_{i}(t)= \begin{cases}b_{\mathrm{inf}} & \text { if } s_{j}(t)=\text { cooperate } \\ b_{\mathrm{inf}}-c_{\mathrm{srv}} & \text { if } s_{j}(t)=\text { defect } \\ -c_{\mathrm{com}} & \text { if } s_{i}(t)=\text { cooperate } \\ 0 & \text { if } s_{i}(t)=\text { defect }\end{cases}
$$

Agent $i$ uses the same utility and strategy functions as its peer $j$, knows the action chosen by $j$ in the current game stage, but it does not record $j$ 's past moves. The ability to memorize the history of actions played by an agent is unrealistic due to the memory constraints of existing smartphone devices. Hence, $i$ is unable to compute $j$ 's cumulative cooperation effort for all game stages. For this reason, agents $i$ and $j$ have imperfect information about the game state.

\section{Two-Agent Game Analysis}

The goal of this section is to determine under which conditions the threshold strategy introduced previously will yield a cooperative equilibrium. Let $U_{i}$ denote the expected aggregate discounted reward for agent $i$. Agents acting rationally will only cooperate if the expected discounted benefit is greater than the expected discounted cost incurred, as defined next:

$$
U_{i}=\sum_{t=0}^{\infty} \delta^{t}\left(b_{i}(t)-c_{i}(t)\right)>0 .
$$

We now calculate the expected discounted aggregate reward, assuming that agents are chosen randomly at each turn to query the LBS.

Lemma 1. If exactly one agent is chosen at each turn to query the LBS and the probability of being chosen equals $1 / 2$, then agent $i$ 's expected aggregate discounted reward, as a function of the threshold $\alpha$, is given by

$$
U_{i}(\alpha)= \begin{cases}\frac{1}{1-\delta} \cdot \frac{b_{i n f}-c_{\text {com }}}{2} & \text { if } \alpha \leq-1, \\ \frac{1}{1-\delta} \cdot \frac{b_{i n f}-c_{s r v}}{2} & \text { otherwise. }\end{cases}
$$


Proof. The probability that an agent $i$ is chosen to query the LBS is $1 / 2$. If agent $i$ is chosen to query the LBS in turn $k$, it will cooperate with the second agent if $\phi_{i}(k)>\alpha$, incurring a cost of $c_{\text {com }}$, and defect otherwise, receiving no payoff. If agent $j$ is selected to query the LBS in turn $k$, it will cooperate with agent $i$ if $\phi_{j}(k)>\alpha$, resulting in a benefit of $b_{\text {inf }}$ for agent $i$, and defect if $\phi_{j}(k) \leq \alpha$, causing agent $i$ to call the LBS server, yielding a lesser benefit of $b_{\text {inf }}-c_{\text {srv }}$. Let $U_{i}(\alpha)$ denote the expected aggregate discounted reward for agent $i$ as a function of the threshold value $\alpha$. Then $U_{i}(\alpha)$ can be calculated as follows:

$$
\begin{aligned}
U_{i}(\alpha)= & \frac{1}{2} \sum_{t=0}^{\infty} \delta^{t}\left[-c_{\mathrm{com}} \cdot 1_{\left\{\phi_{i}(t)>\alpha\right\}}+0 \cdot 1_{\left\{\phi_{i}(t) \leq \alpha\right\}}\right]+ \\
& \delta^{t}\left[b_{\mathrm{inf}} \cdot 1_{\left\{\phi_{j}(t)>\alpha\right\}}+\left(b_{\mathrm{inf}}-c_{\mathrm{srv}}\right) \cdot 1_{\left\{\phi_{j}(t) \leq \alpha\right\}}\right],
\end{aligned}
$$

where $1_{\text {predicate }}$ is an indicator function yielding 1 each time the predicate is true and zero when the predicate is false.

As we do not know the value of $\phi_{i}(t)$ for the duration of the game, we must calculate the average cumulative cooperation effort $(\bar{\phi})$ based on the average number of times an agent receives data $(\overline{\mathrm{rc}})$ and shares data $(\overline{\operatorname{tr}})$, as follows:

$$
\bar{\phi}=\overline{\mathrm{rc}}-\overline{\mathrm{tr}} .
$$

As the two agents have equal probabilities of being chosen to query the LBS at each turn, we expect that each agent is chosen for $m / 2$ game stages, where $m$ is the length of the game. Additionally, agent $i$ will share data with its peer $j$ with an estimated probability of: $\operatorname{Pr}\{\bar{\phi}>\alpha\}$. Hence, $\overline{\mathrm{rc}}$ and $\overline{\operatorname{tr}}$ are given by

$$
\begin{aligned}
& \overline{\mathrm{rc}}=(m / 2) \cdot \operatorname{Pr}\{\bar{\phi}>\alpha\}, \\
& \overline{\operatorname{tr}}=(m / 2) \cdot \operatorname{Pr}\{\bar{\phi}>\alpha\},
\end{aligned}
$$

implying that the average cumulative cooperation effort is zero: $\bar{\phi}=0$. Using the previous results, we can now estimate the value of $U_{i}(\alpha)$ as follows:

$$
\begin{aligned}
U_{i}(\alpha) & =\frac{1}{2} \sum_{t=0}^{\infty} \delta^{t}\left[\left(b_{\mathrm{inf}}-c_{\mathrm{com}}\right) \cdot 1_{\{\alpha<0\}}+\left(b_{\mathrm{inf}}-c_{\mathrm{srv}}\right) \cdot 1_{\{\alpha \geq 0\}}\right]= \\
& = \begin{cases}\frac{1}{1-\delta} \cdot \frac{b_{\mathrm{inf}}-c_{\mathrm{com}}}{2} & \text { if } \alpha \leq-1, \\
\frac{1}{1-\delta} \cdot \frac{b_{\mathrm{inf}}-c_{\mathrm{srv}}}{2} & \text { otherwise } .\end{cases}
\end{aligned}
$$

In the following theorem we characterize the possible equilibria resulting from the game described in Lemma 1 .

Theorem 1. The game described in Lemma 1 has two Nash equilibria: (i) both agents choose a threshold $\alpha=-1$, ensuring the minimal level of cooperation 
necessary to achieve the maximal payoff and (ii) both agents opt for a threshold of $\alpha \geq 0$, thus defecting throughout the game. Further, the choice of $\alpha=-1$ results in a Pareto optimal equilibrium.

Proof. Agents $i$ and $j$ can opt for a threshold of $\alpha>-1$ or, equivalently, $\alpha \geq 0$, leading to a non-cooperative Nash equilibrium. As the function $\phi_{i}(t)$ is initially zero, it will never be greater than zero, and agents will never cooperate: $\forall_{i, t} \phi_{i}(t) \ngtr 0$. If agent $j$ independently chooses a threshold $\alpha^{\prime}$ smaller than zero, it will cooperate, at most, $-\alpha^{\prime}$ times, incurring a cost proportional to $c_{\text {com }}$.

The choice of $\alpha=-1$ is also a Nash equilibrium as it is the maximal threshold that still allows mutual cooperation between both agents, minimizes the number of times they share data, and maximizes their expected discounted payoffs:

$$
\max U_{i, j}(\alpha)=\frac{1}{1-\delta} \cdot \frac{b_{\mathrm{inf}}-c_{\mathrm{com}}}{2} \quad \text { for } \alpha \leq-1 .
$$

If agent $j$ independently decreases its threshold to $\alpha^{\prime}<\alpha$, it will increase its capacity to cooperate with $i$, potentially incurring a higher sharing cost, proportional to $c_{\mathrm{com}}$, and consequently obtaining a lower payoff than (11). Assuming $j$ chooses, instead, $\alpha^{\prime} \geq 0$, it will always defect and agent $i$ will cooperate, at most, once with $j$, at which time $j$ earns $b_{\text {inf }}$ and $i$ has a cost of $c_{\text {com. }}$. As this event occurs, at most, once for an infinitely repeated game, we do not consider this an advantage for player $j$. Instead, we assume that the game outcome is equivalent to when both agents defect throughout the game.

We now compare the two possible choices of threshold values: $\alpha=-1$ and $\alpha \geq 0$. Clearly, $U_{i}(-1)>U_{i}\left(\alpha^{\prime}\right)$, for $\alpha^{\prime} \geq 0$ and $0 \leq c_{\text {com }}<c_{\text {srv }}<b_{\text {inf }} \leq 1$. Hence, the strategical choice of $\alpha=-1$ Pareto dominates the choice $\alpha \geq 0$. As there is no other strategy that Pareto dominates the choice of $\alpha=-1$, we conclude that this choice by both agents is Pareto optimal.

\section{Multiple-agent Game}

In an effort to match more closely a real interaction between MobiCrowd devices, we now consider a game with multiple agents and represent time as a continuous measure. To analyze the behavior of a large population of agents $(n>>2)$, we alter the epidemic model in 14 to support threshold strategies and we define the rate at which agents switch between states, as shown in Figure2, The letters $\mathcal{K}, \mathcal{I}$, and $\mathcal{R}$ in the state-diagram stand for the three agent roles, seeker $(\mathcal{K})$, informed $(\mathcal{I})$, and removed $(\mathcal{R})$ and the integer suffix next to each letter denotes the cumulative cooperation effort. The Cartesian product of the set of roles and the range of the cumulative cooperation effort generates the full set of agent states: $\mathcal{S}=\Pi_{R} \times \Pi_{\phi}$.

Assume that only seekers are present at the start of the game; so all agents are at state $\mathcal{K}_{0}$ (the cumulative cooperation effort is initially zero). With no informed agents in the vicinity, seekers must acquire regional data via the LBS, following transition $\mathcal{K}_{0} \rightarrow \mathcal{I}_{0}$, in which case their cumulative cooperation effort remains 
constant at zero. These newly informed agents can now cooperate with seekers at state $\mathcal{K}_{0}$. A seeker receiving regional data through an informed peer improves its cumulative cooperation effort by one and then jumps to a higher layer in the state diagram. Whereas, informed agents sharing data suffer a decrement in their cumulative cooperation effort and fall to a lower level in the state diagram.

An informed agent $i$ with the minimum cumulative cooperation level (i.e. that has reached the bottom level in the state chart) does not cooperate with seekers as the condition to cooperate, $\phi_{i}(t)>\phi^{\text {min }}=\alpha$, no longer holds (2). To reach the maximum cumulative cooperation effort, an agent $i$ must act solely as a recipient of information and all its $(n-1)$ peers act as providers of information; agent $i$ 's peers descend to the bottom layer of the diagram and reach the minimum cumulative cooperation effort.

Theorem 2. The cumulative cooperation effort of an agent $i, \phi_{i}(t)$, has a maximum of $\phi^{\text {max }}$ and minimum of $\phi^{\text {min }}$, where

$$
\begin{aligned}
\phi^{\max } & =(1-n) \alpha, \\
\phi^{\min } & =\alpha,
\end{aligned}
$$

for an n-agent game and a common threshold value of $\alpha$.

In other words, there is an upper limit to the amount of cooperation an agent $i$ is forced to provide by following the threshold strategy $s_{i}(t)$, which is proportional to the number of agents $n$ in the game and the threshold value $\alpha$. If agent $i$ 's cooperation effort reaches the maximum value, $\phi_{i}(t)=\phi^{\max }, i$ will cooperate, at most, $\phi^{\max }-\phi^{\min }=-n \alpha$ times, for $\phi_{i}(t)=0, i$ will cooperate, at most, $-\alpha$ times, and if $\phi_{i}(t)=\phi^{\text {min }}, i$ will defect.

Proof. Consider an $n$-agent game and let $\mathcal{G}$ be the group of agents consisting of all but agent $p_{1}$. Hence, the cardinality of $\mathcal{G}$ is given by: $|\mathcal{G}|=n-1$. The cumulative cooperation effort of all agents is initially equal to zero. Each time $t$ the regional data in $p_{1}$ 's cache expires, an agent $p$, satisfying $\left\{p \in \mathcal{G} \mid \phi_{p}(t)>\alpha\right\}$, contacts the LBS server and shares the retrieved data with $p_{1}$, upon $p_{1}$ 's request. This benevolent sharing activity ceases at time $t^{\prime}$ when all agents $p \in \mathcal{G}$ have a cumulative cooperation effort of: $\phi_{p}\left(t^{\prime}\right)=\alpha$. If there was an agent $p^{*} \in \mathcal{G}$ such that $\phi_{p^{*}}\left(t^{\prime}\right)=\alpha+1>\alpha$, then $p^{*}$ could share data with $p_{1}$ once more before defecting. Each agent in $\mathcal{G}$ can, thus, share at most $-\alpha$ data items with $p_{1}$ before defecting. Hence, $p_{1}$ 's cumulative cooperation effort reaches a maximum at time $t^{\prime}$, given by:

$$
\phi_{p_{1}}\left(t^{\prime}\right)=|\mathcal{G}|(-\alpha)=(1-n) \alpha,
$$

and those of $p \in \mathcal{G}$ reach a minimum of

$$
\phi_{p}\left(t^{\prime}\right)=\alpha .
$$

The arrows in the state chart denote the rates at which agents switch between states, where $r_{\text {con }}$ refers to the contact rate between game agents in a region, $1 / r_{\text {srv }}$ is the average waiting time before contacting the LBS server, $1 / r_{\text {inf }}$ is the 
average information lifetime, and finally, $r_{\text {req }}$ is the rate at which MobiCrowd users request information. Here $A(t)$ is proportional to the fraction of informed agents with a cumulative cooperation effort above the minimum $\left(\phi^{\mathrm{min}}\right)$, i.e. the pool of informed agents that can cooperate, and $B(t)$ is proportional to the fraction of seekers with a cumulative cooperation effort below the maximal value $\left(\phi^{\max }\right)$, i.e. the group of seekers capable of querying informed peers. In Table 2 we define the payoffs associated with each state transition.

Table 2. Transition Rates and Payoffs

\begin{tabular}{lllr}
\hline Transition & Pre-condition & Rate & Utility \\
\hline 1) $\mathcal{K}_{\phi} \rightarrow \mathcal{I}_{\phi+1}$ & $\phi<\phi^{\max }$ & $A(t) \cdot K_{\phi}(t)$ & $b_{\text {inf }}$ \\
2) $\mathcal{K}_{\phi} \rightarrow \mathcal{I}_{\phi}$ & - & $r_{\text {srv }} \cdot K_{\phi}(t)$ & $b_{\text {inf }}-c_{\text {srv }}$ \\
3) $\mathcal{I}_{\phi+1} \rightarrow \mathcal{I}_{\phi}$ & $\phi<\phi^{\max }$ & $B(t) \cdot I_{\phi+1}(t)$ & $-c_{\text {com }}$ \\
4) $\mathcal{I}_{\phi} \rightarrow \mathcal{R}_{\phi}$ & - & $r_{\text {inf }} \cdot I_{\phi}(t)$ & 0 \\
5) $\mathcal{R}_{\phi} \rightarrow \mathcal{K}_{\phi}$ & - & $r_{\text {req }} \cdot R_{\phi}(t)$ & 0 \\
\hline
\end{tabular}

where:

$$
A(t)=r_{\mathrm{con}} \sum_{l=\phi^{\mathrm{min}}+1}^{\phi^{\max }} I_{l}(t) \text { and } B(t)=r_{\mathrm{con}} \sum_{l=\phi^{\mathrm{min}}}^{\phi^{\mathrm{max}}-1} K_{l}(t) .
$$

When a seeker successfully queries an informed agent, it becomes informed, improves its cumulative cooperation effort by 1 and obtains a benefit of $b_{\text {inf }}$ as defined by Transition 1 . Note that only informed agents with a cumulative cooperation effort greater than $\phi^{\text {min }}$ (or $\alpha$ ) will cooperate with seekers. By following Transition 2, seekers can become informed by connecting to the LBS instead of communicating with a peer. However, in this case, they receive a payoff of just $b_{\text {inf }}-c_{\text {srv }}$ and their cumulative cooperation efforts remain constant. When a seeker $i$ reaches the highest cumulative cooperation effort, $\phi_{i}(t)=\phi^{\max }$, it can only become informed through the LBS server, by following Transition 2 , as there is no other agent $j$ available such that $\phi_{j}(t)>\alpha$ (see proof of Theorem 2). Each time informed agents share data with a seeker, their cumulative cooperation effort drops by 1 unit and they incur a communication cost of $c_{\text {com }}$, according to Transition 3. Finally, the remaining two transitions, 4 and 5, define the rates at which agents switch from the informed to the removed state and from the removed to the seeker state, respectively.

As we define the transition rates between agent states for the $n$-agent game, we ensure that the total information shared is equal to the total amount of information received by seekers. Even though agents are constantly updating the regional data stored in their mobile proxies, this property must still hold because each fresh data item shared by an informed agent must be received by a seeker. This property implies that there is no loss of information during the data sharing process between informed agents and seekers; we assume lost packets are retransmitted. This concept is expressed formally in the following theorem. 
Theorem 3. The total amount of information shared by informed agents must equal the total amount of information received by seekers, assuming the information sharing process incurs no loss of data.

Proof. The total rate at which seekers receive data from informed agents equals

$$
\mathrm{rc}_{\text {total }}(\alpha, t)=\sum_{\phi=\alpha}^{(1-n) \alpha-1} K_{\phi}(t) \cdot r_{\text {con }}\left(I_{\alpha+1}(t)+\ldots+I_{(1-n) \alpha}(t)\right) .
$$

A seeker agent $i$ at state $\mathcal{K}_{\phi^{\max }}$ cannot receive data from an informed agent because state $\mathcal{K}_{\phi^{\max }}$ is only reached when all other agents $j$ have the lowest possible cumulative cooperation effort, $\forall_{j} \phi_{j}(t)=\alpha$, as explained in the proof of Theorem 2, hence any informed agent $j \in \mathcal{I}_{\phi^{\min }}$ following threshold strategy $s_{j}(t)$ will defect.

Similarly, the total rate at which informed agents share data with seekers is given by the following:

$$
\operatorname{tr}_{\text {total }}(\alpha, t)=\sum_{\phi=\alpha+1}^{(1-n) \alpha} I_{\phi}(t) \cdot r_{\text {con }}\left(K_{\alpha}(t)+\ldots+K_{(1-n) \alpha-1}(t)\right) .
$$

Expanding $\mathrm{rc}_{\text {total }}(\alpha, t)$ gives

$$
\begin{aligned}
& \operatorname{rc}_{\text {total }}(\alpha, t)=K_{\alpha}(t) \cdot r_{\text {con }}\left(I_{\alpha+1}(t)+\ldots+I_{(1-n) \alpha}(t)\right)+\ldots \\
& \quad+K_{(1-n) \alpha-1}(t) \cdot r_{\text {con }}\left(I_{\alpha+1}(t)+\ldots+I_{(1-n) \alpha}(t)\right) .
\end{aligned}
$$

By factorizing (16) in terms of $I_{\phi}(t)$, for $\alpha<\phi \leq(1-n) \alpha$, we obtain

$$
\begin{aligned}
& I_{\alpha+1}(t) \cdot r_{\mathrm{con}}\left(K_{\alpha}(t)+\ldots+K_{(1-n) \alpha-1}(t)\right)+\ldots \\
& \quad+I_{(1-n) \alpha}(t) \cdot r_{\mathrm{con}}\left(K_{\alpha}(t)+\ldots+K_{(1-n) \alpha-1}(t)\right)=\operatorname{tr}_{\text {total }}(\alpha, t) .
\end{aligned}
$$

As both rates are equal, $\operatorname{rc}_{\text {total }}(\alpha, t)=\operatorname{tr}_{\text {total }}(\alpha, t)$, the total amount of information shared must equal the total amount of information received:

$$
\int_{0}^{\infty} \mathrm{rc}_{\text {total }}(\alpha, t) \mathrm{d} t=\int_{0}^{\infty} \operatorname{tr}_{\text {total }}(\alpha, t) \mathrm{d} t .
$$

To calculate the total discounted game payoff, we map each transition to a utility value (see Table 21). We assign the highest payoff of $b_{\text {inf }}$ to transition $\mathcal{K}_{\phi} \rightarrow \mathcal{I}_{\phi+1}$, through which seekers acquire regional data from an informed peer. Seekers earn a lesser payoff of $b_{\text {inf }}-c_{\text {srv }}$ per query when they acquire data from the LBS using transition $\mathcal{K}_{\phi} \rightarrow \mathcal{I}_{\phi}$ due to the privacy loss incurred. Informed agents suffer a penalty of $c_{\text {com }}$ whenever they share data with seekers using transition $\mathcal{I}_{\phi+1} \rightarrow \mathcal{I}_{\phi}$. By multiplying the transition rates and the transition utility values we obtain the game's payoff rate $u(\alpha, t)$ (see Appendix A). Applying the discount factor $\delta$ to the payoff rate $u(\alpha, t)$ and integrating the result over the whole 
duration of the game gives the total game discounted reward; in other words, the payoff accumulated by the whole agent population:

$$
U_{\text {total }}(\alpha)=\int_{0}^{\infty} \delta^{t} u(\alpha, t) \mathrm{d} t .
$$

For a finite population of agents $n$, the expected total discounted payoff of each agent is calculated simply as

$$
U(\alpha)=\frac{1}{n} \cdot U_{\text {total }}(\alpha) .
$$

\section{Multiple-agent Game Analysis}

The quantity of agents in each state is controlled by a system of non-linear differential equations (see Appendix B), derived from the agent state-chart in Figure 2. Ideally, the exact expressions for $K_{\phi}(t), I_{\phi}(t)$, and $R_{\phi}(t)$ could be found by solving the system of equations. Unfortunately, the basic W. Kermack and A. McKendrick SIR model, on which our work relies, cannot be solved analytically [6] $]^{1}$. The additional complexity of our own system of differential equations only lessens the chances of finding an analytical solution. However, we can still solve the system of differential equations by using numerical methods [6]. We resort to a numerical ODE solver in Mathematica to compute functions $K_{\phi}(t), I_{\phi}(t)$, and $R_{\phi}(t)$, to analyze the game's evolution and its steady-state equilibrium.

Our first concern in the game analysis is to study the evolution of the rates at which (i) seekers become informed by contacting informed peers $\left(\mathcal{K}_{\phi} \rightarrow \mathcal{I}_{\phi+1}\right)$, (ii) informed agents share data with seekers $\left(\mathcal{I}_{\phi+1} \rightarrow \mathcal{I}_{\phi}\right)$, and (iii) the rate at which seekers become informed by contacting the LBS $\left(\mathcal{K}_{\phi} \rightarrow \mathcal{I}_{\phi}\right)$. We do this because these are the only three transitions that have utilities different from zero (see Table 2). From the plots in Figure 3, it is clear that the rate at which informed agents share data equals the rate at which seekers obtain data from informed peers (plots of $\mathcal{K}_{\phi} \rightarrow \mathcal{I}_{\phi+1}$ overlap those of $\mathcal{I}_{\phi+1} \rightarrow \mathcal{I}_{\phi}$ in 3a and 3d). Varying the contact rate, $r_{\text {con }}$, average information lifetime, $1 / r_{\text {inf }}$, average time before calling the server, $1 / r_{\mathrm{srv}}$, and the request rate, $r_{\text {req }}$, affects the proportion of agents occupying each of the three roles (i.e. seeker, informed, and removed) at equilibrium. By balancing the transition rates (Figure 3d), it is possible to achieve a uniform proportion of agents in each of the three roles (Figure 3e).

Decreasing the common threshold $\alpha$ increases the fraction of informed agents willing to cooperate, i.e. all informed agents $i$ whose cumulative cooperation effort is above the threshold $\phi_{i}(t)>\phi^{\min }=\alpha$. Consequently, seekers begin querying informed peers $\left(\mathcal{K}_{\phi} \rightarrow \mathcal{I}_{\phi+1}\right)$ more often than the LBS $\left(\mathcal{K}_{\phi} \rightarrow \mathcal{I}_{\phi}\right)$, thus raising their payoffs from $b_{\text {inf }}-c_{\text {srv }}$ to $b_{\text {inf }}$ per query. The total discounted payoff stabilizes when the cumulative cooperation effort of most informed agents is above the threshold and the rate of transition $\mathcal{K}_{\phi} \rightarrow \mathcal{I}_{\phi+1}$ no longer increases.

\footnotetext{
${ }^{1}$ See Section 1.5 of [6].
} 


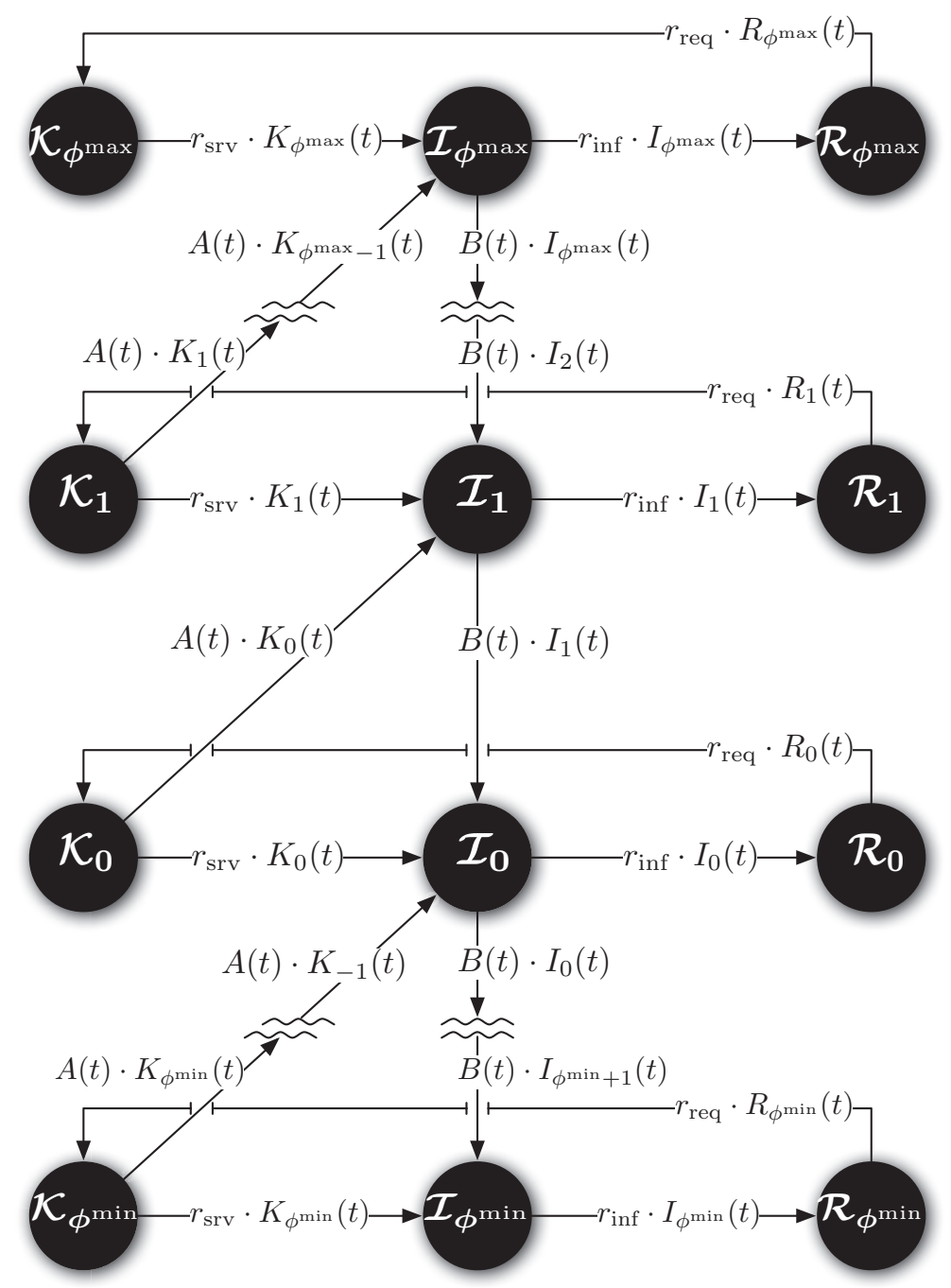

Fig. 2. State diagram of the $n$-agent game. Initially all agents have a null cumulative cooperation effort and are spread out across the three states: $\mathcal{K}_{0}, \mathcal{I}_{0}$, and $\mathcal{R}_{0}$. A seeker at state $\mathcal{K}_{0}$ can become informed by contacting an informed agent at states $\mathcal{I}_{\phi^{\min }+1}, \ldots, \mathcal{I}_{0}, \ldots, \mathcal{I}_{\phi^{\max }}$ and switch to state $\mathcal{I}_{1}$. If this fails, the same seeker can obtain regional data through the LBS, maintaining its cumulative cooperation effort, and switch to state $\mathcal{I}_{0}$. Informed agents at states $\mathcal{I}_{\phi^{\min }+1}, \ldots, \mathcal{I}_{0}, \ldots, \mathcal{I}_{\phi^{\max }}$ can share data with seekers, in which case their cumulative cooperation effort drops by one unit, and they fall to a lower level in the diagram. An informed agent at state $\mathcal{I}_{\phi^{\text {min }}}$ does not cooperate with seekers and seekers at state $\mathcal{K}_{\phi^{\max }}$ can only become informed via the LBS. Once the information expires, informed agents become removed and can later become seekers. The transitions represent the rates at which agents change state. 
This phenomenon is illustrated in Figures $3 \mathrm{C}$ and $3 \mathrm{ff}$, where the total discounted payoff function $U_{\text {total }}(\alpha)$ is almost level for $\alpha \leq-4$ in $3 \mathrm{c}$ and $\alpha \leq-5$ in $3 \mathrm{f}$

We proceed to estimate the distribution of agents for the whole range of cumulative cooperation values, i.e. $\phi \in\left[\phi^{\min }, \phi^{\max }\right]=[\alpha,(1-n) \alpha]$, assuming a uniform distribution of agents for the three possible roles at the game's steady state equilibrium. As we represent the population of agents in each state as a fraction of unity and given that agents can only be in a single state at a time, the sum $K_{\phi}(t)+I_{\phi}(t)+R_{\phi}(t)$ can be interpreted as the probability that a given agent $i$ has a cumulative cooperation effort equal to $\phi$ at time $t$ :

$$
\operatorname{Pr}\left\{\exists_{i}: \phi_{i}(t)=\phi\right\}=K_{\phi}(t)+I_{\phi}(t)+R_{\phi}(t) .
$$

For a large population of agents $(n>>2)$ and at the game's steady-state equilibrium $\left(t \rightarrow \infty\right.$ or $\left.t_{\infty}\right)$, we estimate this probability (21) as

$$
\operatorname{Pr}\left\{\exists_{i}: \phi_{i}\left(t_{\infty}\right)=\phi\right\} \approx \frac{1}{1-\alpha} \cdot \frac{1}{(1-1 / \alpha)^{\phi-\alpha}},
$$

where $\sum_{\phi=\alpha}^{(1-n) \alpha} \frac{1}{1-\alpha} \cdot \frac{1}{(1-1 / \alpha)^{\phi-\alpha}} \rightarrow 1$, for $\alpha \leq-1$ and $n \rightarrow \infty$. In Figure 4 we plot (21) and (22) for a population of $n=40$ agents and a threshold of $\alpha=-2$. The estimate (22) is particularly relevant to characterize agents' rational behavior at equilibrium, which allows them to quantify the rates of all transitions with utilities different from zero, and to be able to choose the optimal threshold $\alpha^{\text {opt }}$ to maximize their payoff.

Assuming we reach a uniform distribution of agents occupying each of the three roles at the game's steady-state equilibrium, as with the case illustrated in Figure 3e, we can estimate the probability that a given agent $i$ is in, for example, state $\mathcal{K}_{\phi}$ as: $1 / 3 \cdot \operatorname{Pr}\left\{\exists_{i}: \phi_{i}\left(t_{\infty}\right)=\phi\right\}$ (the same holds for $\mathcal{I}_{\phi}$ and $\mathcal{R}_{\phi}$ ). This enables us to estimate the total rate at which information is shared by informed agents, $\widehat{\operatorname{tr}}_{\text {total }}(\alpha) \approx \operatorname{tr}_{\text {total }}\left(\alpha, t_{\infty}\right)$, received by seekers contacting informed peers, $\widehat{\mathrm{rc}}_{\text {total }}(\alpha) \approx \mathrm{rc}_{\text {total }}\left(\alpha, t_{\infty}\right)$, and received by seekers contacting the LBS, $\widehat{\mathrm{sv}}_{\text {total }}(\alpha)$, at the game's steady-state equilibrium, as shown below:

$$
\begin{aligned}
& \widehat{\operatorname{tr}}(\alpha)=\frac{r_{\text {con }}}{3^{2}} \cdot \frac{\alpha}{\alpha-1}\left[\left(\frac{\alpha-1}{\alpha}\right)^{n \alpha}-1\right]^{2}, \\
& \widehat{\operatorname{rc}}(\alpha)=\widehat{\operatorname{tr}}(\alpha), \\
& \widehat{\operatorname{sv}}(\alpha)=\frac{r_{\mathrm{srv}}}{3}\left[1-\left(\frac{\alpha-1}{\alpha}\right)^{n \alpha-1}\right] .
\end{aligned}
$$

Using (23)-(25) we estimate the game's total payoff at equilibrium as

$$
\widehat{U}_{\text {total }}(\alpha)=b_{\text {inf }} \cdot \widehat{\mathrm{rc}}(\alpha)-c_{\text {com }} \cdot \widehat{\operatorname{tr}}(\alpha)+\left(b_{\text {inf }}-c_{\text {srv }}\right) \cdot \widehat{\mathrm{sv}}(\alpha)
$$

and an agent $i$ 's total payoff at equilibrium as

$$
\widehat{U}(\alpha)=\frac{1}{n} \cdot \widehat{U}_{\text {total }}(\alpha),
$$

for a large, but finite agent population of size $n(n>>2)$. 
HoKed pejunoos!a Ietol

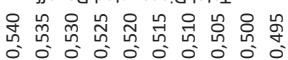
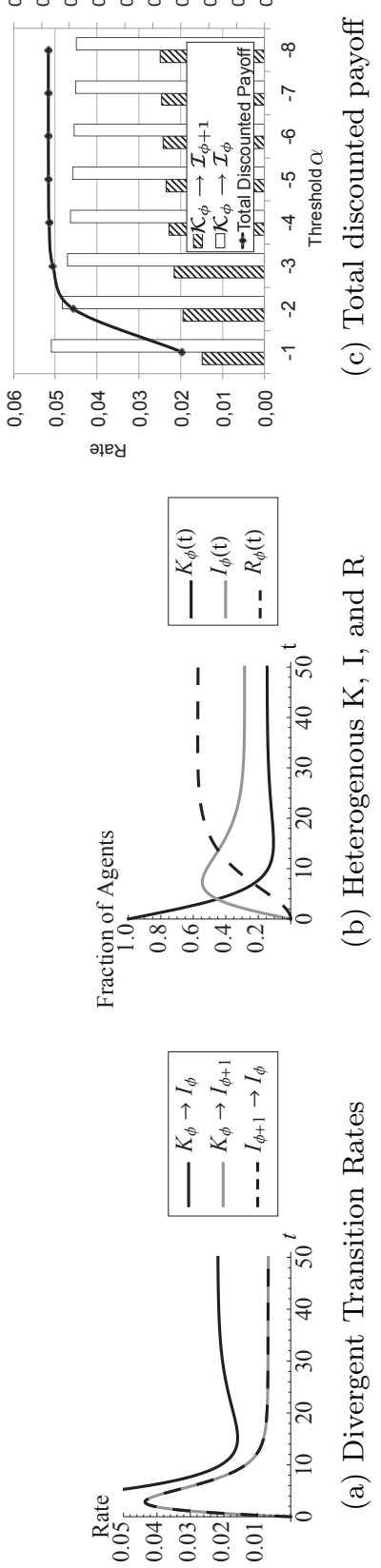

HOKe d pəzunoss!a lę이

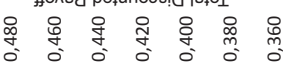

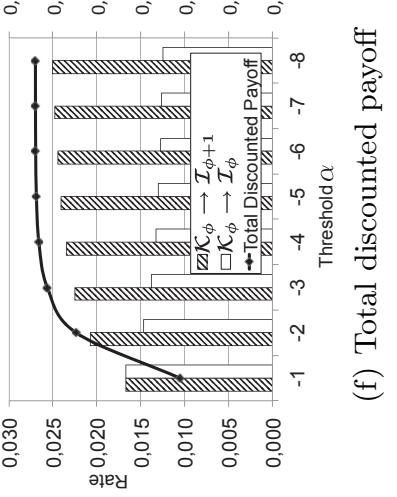

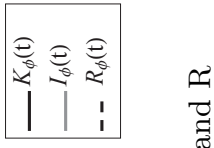
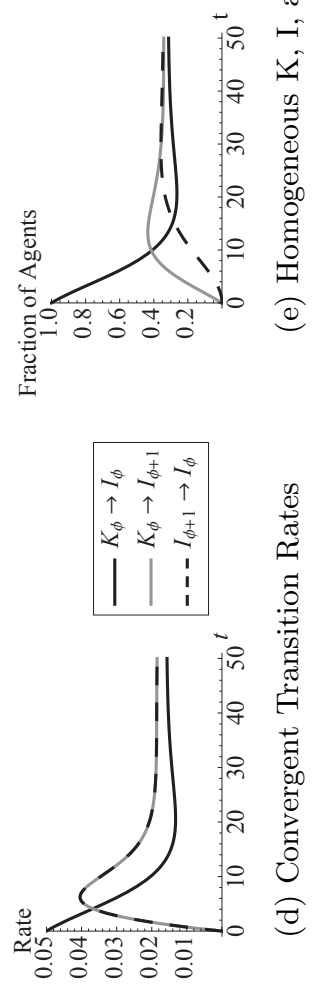

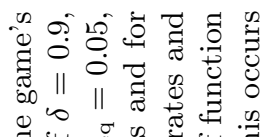

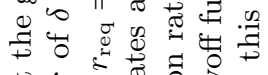

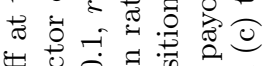

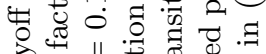

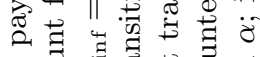

ठ ठ

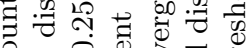
ర) ป สี $\mid 1$ 융 ○ी

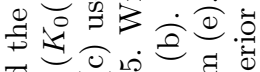
चี

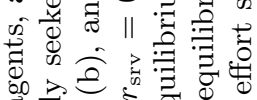
की હ્. ठ․ ठี้

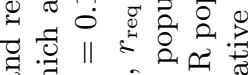

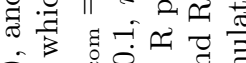
$\hat{E}$

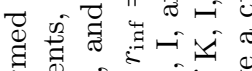

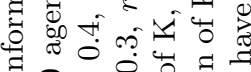
$\exists$ 아 $\|$ || 纯 $\|$ 它

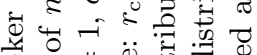

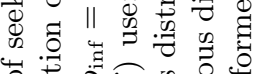

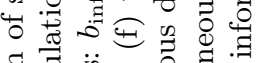

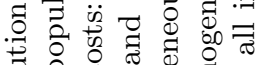

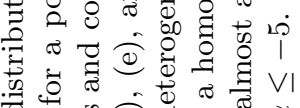
包

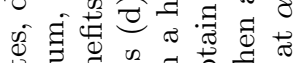

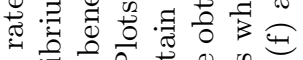

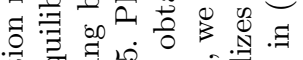

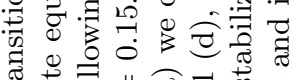

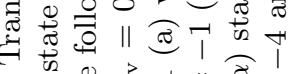

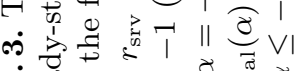

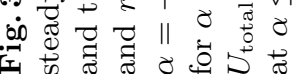




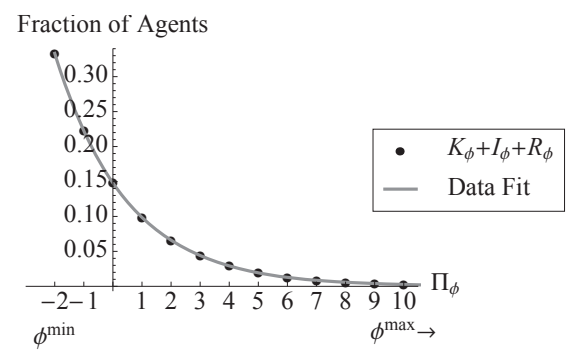

Fig. 4. Distribution of the cumulative cooperation effort. The plot shows the fraction of agents with a specific cumulative cooperation effort, for $\Pi_{\phi}=\left[\phi^{\min }, \phi^{\max }\right]$, assuming we have $1 / 3$ of each type of agent at the game's steady-state equilibrium.

Agents acting to maximize their payoffs will benefit by decreasing their threshold. A lower threshold implies that more agents are willing to cooperate when informed. Seekers can obtain regional data from this larger pool of cooperative informed agents, instead of using the LBS and gathering a higher payoff. Clearly, the benefit of acquiring data from an informed peer and sharing it with another agent is greater than the payoff achieved when connecting to the LBS: $b_{\text {inf }}-c_{\text {com }}>b_{\text {inf }}-c_{\text {srv }}$, provided $0 \leq c_{\text {com }}<c_{\text {srv }}<b_{\text {inf }} \leq 1$. In order to consider an optimal threshold $\left(\alpha^{\mathrm{opt}}\right)$, as illustrated in Figures 3c and 3f. we define a tolerance $\epsilon>0$ such that an agent $i$ will not ponder reducing $\alpha$ to maximize its payoff if the added benefit is less than $\epsilon$ :

$$
\alpha^{\mathrm{opt}}=\max \{\alpha \leq-1: \widehat{U}(\alpha-1)-\widehat{U}(\alpha)<\epsilon\} .
$$

\section{Conclusion}

The MobiCrowd system architecture introduces only minor changes to the way traditional location-based schemes operate, thus enabling users to obtain geographic data from other peers and to potentially improve their location privacy by reducing the amount of queries that participants send to the LBS server [14]. However, the improvement in privacy is only possible if users are willing to cooperate. The goal of our work is to learn if users adopting the MobiCrowd system would be willing to collaborate by sharing regional data between themselves in order to avoid the privacy threat of connecting directly to the LBS provider. To study this problem we first developed a game-theoretic framework, from which we defined two infinitely repeated games of imperfect information.

In the first game, we model two agents that are chosen to query the LBS with equal probability. We derived two Nash equilibria in this game, one favors mutual cooperation that is Pareto optimal and the second favors mutual defection. In the second game, by modifying the original MobiCrowd SIR model [14 to support agent rational behavior using threshold strategies we represent an interaction between multiple agents confined to a single region. Due to the complexity of 
modeling peer-to-peer interactions in this case, we use transition rates to define the pace at which agents change state over time and we assign payoffs to each transition. From the analysis of this game, we derive the optimal threshold that maximizes an agent's expected payoff, for a large population of agents, assuming a uniform distribution of agents at the steady-state equilibrium.

Our results show that rational agents attempting to maximize their payoffs will benefit by sharing data with their peers, both in the controlled environment of the two-agent game and in the more realistic $n$-agent scenario. As a future development of this work, we plan to analyze the same contextualdata sharing problem considering threshold strategies with an independent (per agent) threshold, experiment with different types of reactive strategies, and introduce statistical processes describing the rate at which agents enter and leave a region.

\section{References}

1. Alpcan, T.: Network Security: A Decision and Game-Theoretic Approach. Cambridge University Press (2010)

2. Başar, T., Olsder, G.: Dynamic noncooperative game theory. Society for Industrial Mathematics (1999)

3. Bauch, C., Earn, D.: Vaccination and the theory of games. Proceedings of the National Academy of Sciences of the United States of America 101(36), 13391 (2004)

4. Buttyan, L., Hubaux, J.P.: Security and Cooperation in Wireless Networks: Thwarting Malicious and Selfish Behavior in the Age of Ubiquitous Computing. Cambridge University Press, New York (2007)

5. Dhar, S., Varshney, U.: Challenges and business models for mobile locationbased services and advertising. Commun. ACM 54, 121-128 (2011), http://doi.acm.org/10.1145/1941487.1941515

6. Earn, D.: A Light Introduction to Modelling Recurrent Epidemics. In: Brauer, F., van den Driessche, P., Wu, J. (eds.) Mathematical Epidemiology. Lecture Notes in Mathematics, vol. 1945, pp. 3-17. Springer, Heidelberg (2008)

7. Felegyhazi, M., Hubaux, J., Buttyan, L.: Nash equilibria of packet forwarding strategies in wireless ad hoc networks. IEEE Transactions on Mobile Computing 5(5), 463-476 (2006)

8. Freudiger, J., Manshaei, M.H., Hubaux, J.P., Parkes, D.C.: On Non-cooperative Location Privacy: A Game-theoretic Analysis. In: ACM Conference on Computer and Communications Security (CCS) (2009), http://www.sigsac.org/ccs/CCS2009/

9. Humbert, M., Manshaei, M.H., Freudiger, J., Hubaux, J.-P.: Tracking Games in Mobile Networks. In: Alpcan, T., Buttyán, L., Baras, J.S. (eds.) GameSec 2010. LNCS, vol. 6442, pp. 38-57. Springer, Heidelberg (2010)

10. Krumm, J.: A survey of computational location privacy. Personal and Ubiquitous Computing 13(6), 391-399 (2009)

11. Manshaei, M., Zhu, Q., Alpcan, T., Basar, T., Hubaux, J.P.: Game Theory Meets Network Security and Privacy. Tech. rep., École Polytechnique Fédérale de Lausanne, Lausanne (2010) 
12. Olumofin, F., Tysowski, P.K., Goldberg, I., Hengartner, U.: Achieving Efficient Query Privacy for Location Based Services. In: Atallah, M.J., Hopper, N.J. (eds.) PETS 2010. LNCS, vol. 6205, pp. 93-110. Springer, Heidelberg (2010)

13. Osborne, M., Rubinstein, A.: A Course in Game Theory. The MIT press (1994)

14. Shokri, R., Papadimitratos, P., Theodorakopoulos, G., Hubaux, J.P.: Collaborative Location Privacy. In: 2011 IEEE 8th International Conference on Mobile Adhoc and Sensor Systems, MASS (2011)

15. Theodorakopoulos, G., Le Boudec, J.Y., Baras, J.S.: Selfish Response to Epidemic Propagation. IEEE Transactions on Automatic Control (2010)

\section{Appendix A Total Discounted Payoff}

Using the transitions, rates and payoffs (defined in Table 2), we can calculate the total discounted game payoff by integrating over the duration of the game the product of the payoff rate, $u(\alpha, t)$, and the discount factor, $\delta \in] 0,1[$. The payoff rate, $u(\alpha, t)$, is defined as the difference between the benefit rate $b(\alpha, t)$, and the cost rate, $c(\alpha, t)$ :

$$
u(\alpha, t)=b(\alpha, t)-c(\alpha, t)
$$

where

$$
b(\alpha, t)=b_{\mathrm{inf}} \sum_{l=\alpha}^{(1-n) \alpha-1} A(t) \cdot K_{l}(t)+\left(b_{\mathrm{inf}}-c_{\mathrm{srv}}\right) \sum_{l=\alpha}^{(1-n) \alpha} r_{\mathrm{srv}} \cdot K_{l}(t),
$$

and

$$
c(\alpha, t)=c_{\mathrm{com}} \sum_{l=\alpha+1}^{(1-n) \alpha} B(t) \cdot K_{l}(t) .
$$

We now apply Definition 2 to calculate the total discounted reward as a function of the threshold $\alpha$ :

$$
U_{\text {total }}(\alpha)=\int_{0}^{\infty} \delta^{t} u(\alpha, t) \mathrm{d} t
$$




\section{Appendix B Game Dynamics}

The system of non-linear differential equations governing the fraction of agents in each state, $K_{\phi}(t), I_{\phi}(t), R_{\phi}(t)$, for $\phi \in\left[\phi^{\min }, \phi^{\max }\right]$, is

$$
\begin{aligned}
K_{\phi^{\max }}^{\prime}(t) & =r_{\mathrm{req}} \cdot R_{\phi^{\max }}(t)-r_{\mathrm{srv}} \cdot K_{\phi^{\max }}(t) \\
I_{\phi^{\max }}^{\prime}(t) & =A(t) \cdot K_{\phi^{\max }-1}(t)+r_{\mathrm{srv}} \cdot K_{\phi^{\max }}(t) \\
& -\left(B(t)+r_{\mathrm{inf}}\right) I_{\phi^{\max }}(t) \\
R_{\phi^{\max }}^{\prime}(t) & =r_{\mathrm{inf}} \cdot I_{\phi^{\max }}(t)-r_{\mathrm{req}} \cdot R_{\phi^{\max }}(t) \\
K_{\phi^{\min }<\phi<\phi^{\max }}^{\prime}(t) & =r_{\mathrm{req}} \cdot R_{\phi}(t)-\left(A(t)+r_{\mathrm{srv}}\right) K_{\phi}(t) \\
I_{\phi^{\min }}^{\prime}<\phi<\phi^{\max } & (t) \\
& =A(t) \cdot K_{\phi-1}(t)+r_{\mathrm{srv}} \cdot K_{\phi}(t)-\left(B(t)+r_{\mathrm{inf}}\right) I_{\phi}(t) \\
& +B(t) \cdot I_{\phi+1}(t) \\
R_{\phi^{\min }}^{\prime}<\phi<\phi^{\max } & =r_{\mathrm{inf}} \cdot I_{\phi}(t)-r_{\mathrm{req}} \cdot R_{\phi}(t) \\
K_{\phi^{\min }}^{\prime}(t) & =r_{\mathrm{req}} \cdot R_{\phi^{\min }}(t)-\left(A(t)+r_{\mathrm{srv}}\right) K_{\phi^{\min }}(t) \\
I_{\phi^{\min }}^{\prime}(t) & =r_{\mathrm{srv}} \cdot K_{\phi^{\min }}(t)+B(t) \cdot I_{\phi^{\min }+1}(t)-r_{\mathrm{inf}} \cdot I_{\phi^{\min }}(t) \\
R_{\phi^{\min }}^{\prime}(t) & =r_{\mathrm{inf}} \cdot I_{\phi^{\min }}(t)-r_{\mathrm{req}} \cdot R_{\phi^{\min }}(t)
\end{aligned}
$$

As agents can only be in a single state at a time, the following relationship must also hold:

$$
\sum_{l=\phi^{\min }}^{\phi^{\max }} K_{l}(t)+\sum_{l=\phi^{\min }}^{\phi^{\max }} I_{l}(t)+\sum_{l=\phi^{\min }}^{\phi^{\max }} R_{l}(t)=1 .
$$

